\title{
Microscopic Study of Defect Luminescence between 0.72 - 0.85 eV by Optical Microscopy
}

\author{
Dominik Lausch, Christian Hagendorf \\ Fraunhofer Center for Silicon Photovoltaics CSP, Halle, Germany. \\ Email: dominik.lausch@csp.fraunhofer.de \\ Received November $19^{\text {th }}$, 2013; revised December 21 $1^{\text {st }}$, 2013; accepted January $6^{\text {th }}, 2014$ \\ Copyright (c) 2014 Dominik Lausch, Christian Hagendorf. This is an open access article distributed under the Creative Commons \\ Attribution License, which permits unrestricted use, distribution, and reproduction in any medium, provided the original work is \\ properly cited. In accordance of the Creative Commons Attribution License all Copyrights (C) 2014 are reserved for SCIRP and the \\ owner of the intellectual property Dominik Lausch, Christian Hagendorf. All Copyright (C 2014 are guarded by law and by SCIRP as \\ a guardian.
}

\begin{abstract}
In this contribution, an experimental setup to investigate the defect luminescence between $0.72-0.85 \mathrm{eV}$ of single defects in Silicon by optical microscopy is introduced. For this purpose, an optical microscope is equipped with an InGaAs CCD detector and a longpass filter with a cut-off wavelength at $1450 \mathrm{~nm}$ in order to filter out the band-to-band luminescence at around $1.1 \mathrm{eV}$. Grain boundaries showing homogeneous distributed defect luminescence can be localized at a $\mu \mathrm{m}$-scale.
\end{abstract}

\section{KEYWORDS}

\section{Defect Luminescence; Recombination Active Defects; Silicon Solar Cells; Optical Microscopy}

\section{Introduction}

Currently, the main challenges of the photovoltaic industry are to reduce the costs per watt peak and to increase the durability of the solar modules. To reduce the costs, a way is to use multicrystalline silicon (mc-Si) wafers. However, the efficiency of solar cells made of mc-Si wafers is lower compared to solar cells made of monocrystalline silicon wafers mainly caused by recombination active defects. These defects have an influence on: 1) the solar-cell efficiency [1-3] and 2) the electrical breakdown behavior hence the reliability of the solar module [4]. Therefore, it is of utmost importance to understand the cause and the physical mechanisms taking place at these defect structures. For this electroluminescence imaging (EL) is a suitable way since it combines various advantages, i.e. inexpensive and reliable equipment supplying a high throughput as well as spatially resolved information [5]. It was shown that mc-Si solar cells suffer from a large number of different recombination active defects. In order to distinguish and investigate these defects at the nm-scale, a classification applicable on a macroscopic (solar-cell level) and a microscopic scale ( $\mu \mathrm{m}-$ scale) were introduced [1]. It was shown that recombina- tion active defects can be separated on solar-cell level into type-A and type-B defects. Type-B defects are mainly characterized by an increased defect luminescence between $0.72-0.85 \mathrm{eV}$. To investigate these types of defects, the defect luminescence of single crystal defects was localized on a $\mu \mathrm{m}$-scale by optical microscopy.

Defect luminescence imaging on a solar-cell level was already observed by diverse authors [6-11]. In 2007, Trupke et al. [6] have shown an increased ELsub intensity at defects presented in string-ribbon based solar-cells. The cause of this defect luminescence of Si solar cells was discussed by many authors justifying the occurrence by an increased oxygen concentration [11] or by $\mathrm{SiN}$ layer deposition [9]. Beside a discussion about a possible explanation of the defect luminescence by a recombination via one or two coupled defect levels, Dreckschmidt et al. [12] and D. Mankovics et al. [13] have shown a correlation of the position of increased defect luminescence with regions having grain boundaries in higher lateral resolution by defect luminescence imaging. However, a one to one correlation with single defects and positions with increased defect luminescence was hardly possible or the needed equipment is quite elaborate. 
In this contribution, the experimental setup and the method to investigate the defect luminescence of individual defects by optical microscopy shall be described in more detail. We will present the details of the experimental setup including specifications of the method regarding spatial and spectral resolution. Furthermore, the results of sub-band gap defect luminescence investigations will be presented starting from a solar cell level down to the $\mu \mathrm{m}$-scale. In particular, microscopic luminescence data will be compared to high resolution SEM/ EBIC investigations of the same recombination active defects. This work will focus on methodological details of the investigations. Details of material related defect properties and physical mechanisms are discussed in another publication $[9,11]$.

\section{Experimental}

The samples analyzed were standard screen printed mc-Si solar cells made from eg-Si feedstock produced in an industrial production line. The lateral distribution of recombination active defects is imaged by forward biased spectrally-integrated, spatially-resolved electroluminescence (EL) imaging using a standard setup [5]. All EL images were acquired at room temperature using a $\mathrm{Si}$ charge coupled device (CCD) camera. In order to detect the defect luminescence between $0.72-0.85 \mathrm{eV}$ the setup has been upgraded as described below. High resolution images of recombination active defects are based on electron beam induced current (EBIC) measurements performed with a Hitachi SU 70 equipped with an EBIC system of point electronic.

\section{Prepare Experimental Setup for Defect Luminescence Imaging}

\subsection{Abbreviations Defect Luminescence Imaging on Solar-Cell Level}

The standard EL setup consists in general of a power supply, a contacting unit and a Si CCD camera which is covered by a light-tight black box to ensure dark light condition. In our setup, the EL setup was equipped with an InGaAs CCD as shown schematically in Figure 1(a) (ELsub). The InGaAs camera is a XEVA-FPA-1.7-640 from the company XenICs which is thermoelectric cooled to $263 \mathrm{~K}$ using the Peltier effect. The camera has a spectral band from $900 \mathrm{~nm}$ to $1700 \mathrm{~nm}$ with a lateral resolution of $640 \times 512$ pixel and a pixel pitch of $20 \mu \mathrm{m}$. In order to filter out the band-to-band luminescence at around $1.1 \mathrm{eV}$ a longpass filter with a cut-on wavelength of $1450 \mathrm{~nm}$ was added between the lenses and the InGaAs CCD (green ring in Figure 1(a)). The solar-cell was electrically contacted on the rear side by an aluminum chuck and on the front side grid. A four point measurement was used to ensure the applied voltage and

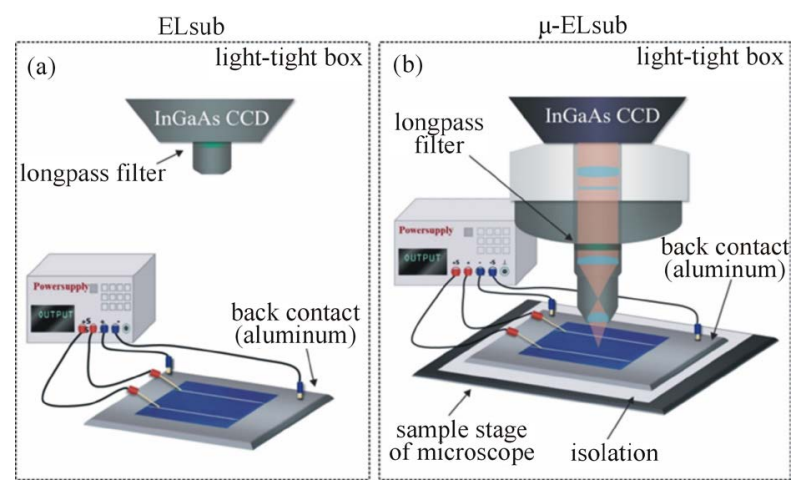

Figure 1. (a) Schema of the ELsub setup. The longpass filter was added in betweens the lenses and the InGaAs camera (green ring). (b) Schema of the $\mu$-ELsub setup. The camera was mounted onto a microscope and the filter was placed into the optical path of the microscope. In both cases the solar cell is contacted by an aluminum chuck (back side) and needles or solder wires (front contact).

measure correctly the current. For our measurements we used a current of $\mathrm{I}=5 \mathrm{~A}$ and voltage of $\mathrm{U} \sim 0.6 \mathrm{~V}$. The integration times were around $10 \mathrm{~s}$ to $30 \mathrm{~s}$ depending on the signal strength. Regions with a high defect luminescence appear bright (see Figure 2(b)). The lateral resolution of the measurements is basically limited by the lower lateral resolution of the InGaAs CCD compared to common Si CCDs and is approximately at around 500 $\mu \mathrm{m}$.

\subsection{Defect Luminescence Imaging on $\mu \mathrm{m}$-Scale}

For investigations on a micrometer scale the InGaAs CCD camera was mounted onto an optical microscope; a Zeiss Axio Scope.A1 ( $\mu$-ELsub). The setup shown in Figure 1(b) is quite similar to the setup for measuring the prebreakdown positions of a solar-cell under reverse bias (micro reverse-biased electroluminescence- $\mu$-ReBEL) as shown in [14]. However, a longpass filter was added to the optical path of the microscope to filter out the band-to-band luminescence of the forward biased solar-cell. The solar-cell was electrically contacted on the scanning stage by and aluminum chuck (back contact) and needles or solder wires (front contact). The forward bias conditions of the solar-cell, hence the current of I = $5 \mathrm{~A}$ and voltage of $\mathrm{U} \sim 0.6 \mathrm{~V}$, were taken according to EL measurements in Section 3.1. In $\mu$-ELsub usually integration times over $60 \mathrm{~s}$ were used. All measurements were performed in reflected light bright field condition and under dark room conditions. However, the different spectrum of about $0.8 \mathrm{eV}$ compared to standard EL at about $1.1 \mathrm{eV}$ leads to a slightly shifted focal plane which was optimized under light. The lateral resolution $d$ is actually limited by the lateral resolution of the microscope which is theoretically given with the wavelength $\lambda$ $=1550 \mathrm{~nm}$ and an aperture angle of $\alpha$ to $\mathrm{d}=0.61 \times \lambda / \sin$ 
$\alpha \sim 1 \mu \mathrm{m}$. However, it will be shown later that the resolution is actually limited by the defect luminescence itself.

\section{Results}

\subsection{Imaging of Defect Luminescence on Solar-Cell Level}

In Figure 2(a), an EL image of a mc-Si solar cell at a current $\mathrm{I}=5 \mathrm{~A}$, voltage $\mathrm{U} \sim 0.6 \mathrm{~V}$ and $1 \mathrm{~s}$ Integration time is shown. Recombination active defects can be localized by their decreased EL signal, hence by a decreased intensity of the band-to-band luminescence at $\sim 1.1 \mathrm{eV}$. The same solar-cell was investigated with the same parameters of the power supply (I and U) by the ELsub setup, but with an integration time of about $60 \mathrm{~s}$ (see Figure 2(b)). The bright regions are region emitting a high intensity of the defect luminescence between 0.72 $0.85 \mathrm{eV}$. Note, the band-to-band luminescence is filtered out. Here, only so called type-B defects (marked with an orange rectangle) appear with a strongly increased intensity of defect luminescence.

Three prominent regions were selected for spectrally resolved EL measurements at room temperature shown in Figure 2(c). The inset in the upper left shows the whole measured spectrum with a high EL intensity at $1.1 \mathrm{eV}$ caused by the band-to-band transition [5]. The dark blue marked region between 0.7 and $1 \mathrm{eV}$ is shown in Figure 2(c). As mentioned type-B defects (orange line in Figure 2(c)) exhibit a high intensity between 0.75 and $0.9 \mathrm{eV}$ compared to the rest of the solar-cell in good agreement to the ELsub imaging in Figure 1(b). Regions with lower or no defect luminescence in Figure 2(b) are showing lower or no defect luminescence in Figure 2(c) respectively. In general, the increased ELsub intensity shows in all investigated positions a broad peak with a maximum at around $0.8 \mathrm{eV}$.

\subsection{Imaging of Defect Luminescence on um-Scale}

In order to achieve a clear one to one correlation of single defects and positions with increased defect luminescence, we have applied an InGaAs CCD detector to an optical microscope (see Section 3.2). In Figure 3, two different regions taken out of regions having an increased intensity of defect luminescence are shown. In the EBIC image in Figures 3(a) and (b), line defects with a decreased EBIC signal indicating an increased recombination activity can be seen. These line defects are small angle grain boundaries (not shown here). Exactly at these grain boundaries an increased intensity of the defect luminescence can be seen by $\mu$-ELsub as bright contrast in Figures 3(c) and (d), hence the grain boundaries can be clearly classified as type-B defects [10]. Few prominent
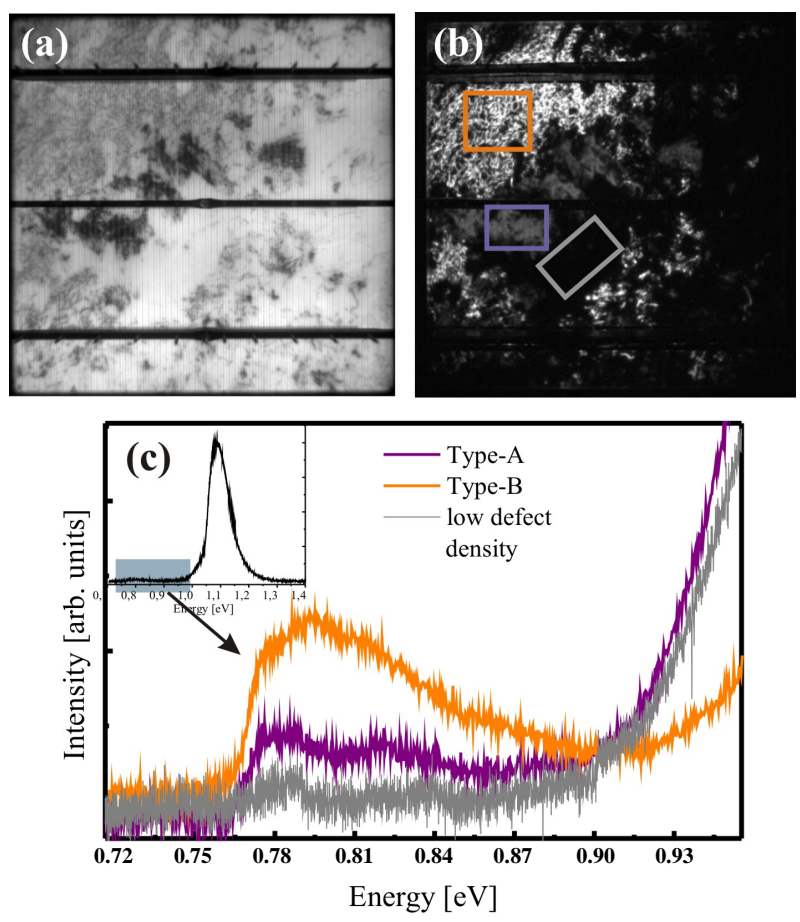

Figure 2. (a) EL and (b) ELsub imaging at $I=5 \mathrm{~A}$ and $U \sim$ $0.6 \mathrm{~V}$. Three prominent regions selected for spectrally resolved EL measurements are marked. (c) Spectrally resolved EL measurements at $I=5 \mathrm{~A}$ and $U \sim 0.6 \mathrm{~V}$. The spectra are colored in dependent of the selected positions. The spectra are not spectrally corrected.
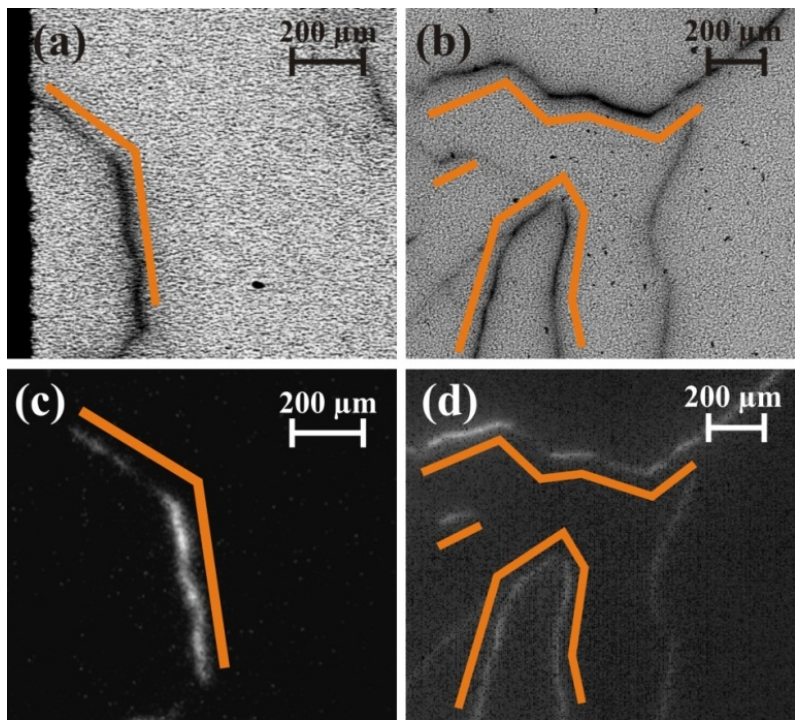

Figure 3. (a), (b) EBIC image of two regions taken out of regions showing an increased intensity of defect luminescence. Here, recombination active line defects (grain boundaries) can be localized by their decreased EBIC signal. (c), (d) $\mu$-ELsub image at $\mathrm{I}=5 \mathrm{~A}$ and $\mathrm{U} \sim 0.6 \mathrm{~V}$ with integration time of about $60 \mathrm{~s}$ of the same region shown in (a) and (b) respectively. The increased defect luminescence at the grain boundaries can be seen by the bright contrast. Few prominent grain boundaries are marked by orange lines. 
grain boundaries are marked by orange lines. The defect luminescence seems to occur homogeneous distributed along the grain boundary rather than spot-like localized, which is in good agreement to results of Schmid et al. [15] who have scanned laterally the so called D1 luminescence at $0.8 \mathrm{eV}$. Nevertheless, the defect luminescence occur a bit blurred around the defect structures limiting the possible lateral resolution strongly. Even under high magnification no localized defect luminescence can be observed, but further measurements will give more knowledge, i.e. varying voltage and temperature, increasing magnification and integration time.

\section{Summarry}

In this contribution, a simple method to locate the defect luminescence between $0.72-0.85 \mathrm{eV}$ by optical microscopy of single defects is introduced. For this, an optical microscope is equipped with an InGaAs CCD detector and a longpass filter with a cut-on wavelength of 1450 $\mathrm{nm}$ in order to filter out the band-to-band luminescence at around $1.1 \mathrm{eV}$. Grain boundaries showing homogeneous distributed defect luminescence can be localized up to a $\mu \mathrm{m}$-scale. This method opens the opportunity for further understanding and improvements in material science.

\section{Acknowledgements}

Susanne Richter is kindly acknowledged for various supports by preparing this manuscript.

\section{REFERENCES}

[1] D. Lausch, K. Petter, B. Henke, R. Bakowskie, S. Schweizer and C. Hagendorf, "Classification of Recombination Active Defect Structures in Multicrystalline Silicon Solar Cells,” Energy Procedia, Vol. 8, 2011, pp. 2834. http://dx.doi.org/10.1016/j.egypro.2011.06.097

[2] H.-J. Möller, "Multicrystalline Silicon for Solar Cells," Solid State Phenomena, Vol. 47-48, 1996, pp. 127-142. http://dx.doi.org/10.4028/www.scientific.net/SSP.47-48.1 $\underline{27}$

[3] S. Kusanagi, T. Sekiguchi, B. Shen and K. Sumino, "Electrical Activity of Extended Defects and Gettering of Metallic Impurities in Silicon,” Materials Science and Technology, Vol. 11, No. 7, 1995, pp. 682-690.

[4] O. Breitenstein, J. Bauer, K. Bothe, W. Kwapil, D. Lausch, U. Rau, J. Schmidt, M. Schneemann, M. C. Schubert, J.-M. Wagner and W. Warta, "Understanding Junction Breakdown in Multicrystalline Solar Cells,” Journal of Applied Physics, Vol. 109, No. 7, 2011, Article ID: 071101. http://dx.doi.org/10.1063/1.3562200
[5] T. Fuyuki, H. Kondo, T. Yamazaki, Y. Takahashi and Y. Uraoka, "Photographic Surveying of Minority Carrier Diffusion Length in Polycrystalline Silicon Solar Cells by Electroluminescence,” Applied Physics Letters, Vol. 86, No. 26, 2005, Article ID: 262108. http://dx.doi.org/10.1063/1.1978979

[6] T. Trupke, et al., "Progress with Luminescence Imaging for the Characterisation of Silicon Wafers and Solar Cells,” 22nd EU-PVSEC, Milan, 2007, pp. 1309-1313.

[7] K. Bothe, K. Ramspeck, D. Hinken, C. Schinke, J. Schmidt, S. Herlufsen, R. Brendel, J. Bauer, J.-M. Wagner, N. Zakharov and O. Breitenstein, "Luminescence Emission from Forward- and Reverse-Biased Multicrystalline Silicon Solar Cells,” Journal of Applied Physics, Vol. 106, No. 10, 2009, Article ID: 104510. http://dx.doi.org/10.1063/1.3256199

[8] M. C. Schubert, et al., "Analysis of Performance Limiting Material Properties of Multicrystalline Silicon," Solar Energy Materials and Solar Cells, Vol. 94, No. 9, 2010, pp. 1451-1456.

http://dx.doi.org/10.1016/j.solmat.2010.04.010

[9] S. Johnston, F. Yan, D. Dorn, K. Zaunbrecher, M. AlJassim, O. Sidelkheir and K. Ounadjela, “Comparison of Photoluminescence Imaging on Starting Multi-Crystalline Silicon Wafers to Finished Cell Performance,” 38th IEEE Photovoltaic Specialists Conference, Austin, Piscataway, 3-8 June 2012, pp. 000406-000410.

[10] D. Lausch, K. Petter, R. Bakowskie, J. Bauer, O. Breitenstein and C. Hagendorf, "Classification and Investigation of Recombination Active Defect in Multicrystalline Silicon Solar-Cells," Proceedings of the 27th EUPVSEC, Frankfurt, 25-28 September 2012, pp. 723-728.

[11] K. Bothe, R. J. Falster and J. D. Murphy, "Room Temperature Sub-Bandgap Photoluminescence from Silicon Containing Oxide Precipitates,” Applied Physics Letters, Vol. 101, No. 3, 2012, pp. 032107-032107-4. http://dx.doi.org/10.1016/j.solmat.2010.04.010

[12] F. Dreckschmidt and H.-J. Möller, "Defect Luminsecence at Grain Boundaries in Multicrystalline Silicon,” Physica Status Solidi (C), Vol. 8, No. 4, 2011, pp. 1356-1360.

[13] D. Mankovics, et al., "Luminescence Emission from Forward- and Reverse-Biased Multicrystalline Silicon Solar Cells,” Physica Status Solidi (A), Vol. 209, No. 10, 2012, p. 1908. http://dx.doi.org/10.1002/pssa.201200133

[14] D. Lausch, K. Petter, H. V. Wenckstern and M. Grundmann, "Correlation of Pre-Breakdown Sites and Bulk Defects in Multicrystalline Silicon Solar Cells," Physica Status Solidi (RRL), Vol. 3, No. 2-3, 2009, pp. 70-72. http://dx.doi.org/10.1002/pssr.200802264

[15] R. P. Schmid, D. Mankovics, T. Arguirov, M. Ratzke, T. Mchedlidze and M. Kittler, "Rapid Dislocation-Related D1-Photoluminescence Imaging of Multicrystalline Si Wafers at Room Temperature," Physica Status Solidi A, Vol. 208, No. 4, 2011, pp. 888-892. http://dx.doi.org/10.1002/pssa.201026269 